\title{
Machine Learning: Object Recognition Web Platform
}

\section{Gupta $\mathrm{AK}^{*}$ and Gupta S}

Apeejay Stya University, Sohna, Gurgaon, Haryana, India

\begin{abstract}
Machine Learning is the backbone and most essential ingredient for working in the field of Artificial Intelligence as well as working in advance technologies. Some of the models which we already using are RNN, SPPNET. In this research paper we will be getting to know about the New Neural Model called Renshaw Model with its advantages and the web platform which makes the life easier for the user to work and understand the neural networks through machine learning. Usage of Cloud services and as well the usage of API will also be defined. The web platform is developed to tackle the issue of complexity storage, and security issue. This platform is basically running in three different formats and according to the user professionality the execution takes place. Researchers in the field of artificial intelligence can contribute and change the dimensions of the execution by designing and executing their code in swagger.api.
\end{abstract}

Keywords: Neural models; Algorithms; Vize; Use cases

\section{Introduction}

When the researchers and the scientists of Defence bodies of India like DRDO were using the already trained models they were facing the issues related to the object detection and hence the time complexity issue was also raised. The blurred images were unable to be recognised as well as the tiny objects in the image which is captured. Sensor technology was also not supported with these models [1]. Before moving ahead to the new proposed model, we should first need to know the terminologies so that it can be simpler for to move ahead with the advanced neural model known as "Renshaw Model".

\section{Machine learning}

The programming concept which provides the life to field of Artificial Intelligence; providing the ability to the system automatically learn and improves from the experience by having the unique result. It focusses on the innovative development in the world of AI by the virtue of computer programs that can access data [2].

The process of learning begins with the observing the data such as the project which has been live working with the collaboration of Campus London and Defence Research Development Organisation which is the defence body of India. Hence looking for patterns in data and make better decisions and reducing the time complexity for improving the accuracy and future results. The primary aim is to provide the ability to the computers to learn automatically without the human intervention. The two types of learning which has been focused and taken into consideration are:

Supervised learning: The goal of supervised learning is to learn patterns from historical data and find similar patterns in new samples. Input data must be annotating often by human. It is the most common machine learning and includes applications like image recognition, object detection and natural language processing.

Unsupervised learning: The goal of unsupervised learning is to find patterns in the set of unlabelled data. The common unsupervised method is cluster analysis and it is used to find hidden patterns or grouping in data. Its Application in computer vision is for example grouping images with similar features or style.

Semi supervised learning: Semi supervised learning is an expensive operation to be performed and executed only by professionals. It provides the dynamic learning. Firstly, we are training the model with the specified set of data and at the lateral stage we are making to work dynamically and detecting the objects, patterns automatically.

\section{Neural Models}

Neural models are based on the concept of neural network which is biologically computer programs that are simulate the way in which the human brain process the data and information. Neural Network Model gathers the knowledge by detecting the patterns and same group in provided data and dynamic data and learn through experience but surely not from programming [3]. It is basically formed from hundreds of single unit neurons connected with coefficients which constitute neural structure and are organised in layers. The study of neural models is divided into two groups:

\section{Trained models}

RCNN: The goal of R-CNN is to take in an image, and correctly identify where the main objects (via a bounding box) in the image. Inputs: Image; Outputs: Bounding boxes + labels for each object in the image. R-CNN creates bounding boxes known as annotating image using a process called Selective Search. At a high level, Selective search looks at the image through different size windows and each size try to group together by texture or intensity to identify objects (Figure 1).

SPPNET: It uses a technique known as RoI Pool (Region of Interest Pooling). At its core, RoIPool shares the forward pass of a CNN for an image across its sub-regions. In the image above, notice how the CNN features for each region are obtained by selecting a corresponding region from the CNN's feature map. Then, the features in each region are pooled (usually using max pooling) (Figure 2).

\section{Proposed model}

Renshaw model: The model which is the solution to most crucial

*Corresponding author: Gupta AK, Apeejay Stya University, Sohna-122 103 Gurgaon, Haryana, India, Tel: +919915966844; E-mail: anitya.gupta320@gmail.com

Received July 07, 2018; Accepted October 12, 2018; Published October 15, 2018

Citation: Gupta AK, Gupta S (2018) Machine Learning: Object Recognition Web Platform. J Comput Sci Syst Biol 11: 306-309. doi:10.4172/jcsb.1000289

Copyright: ( 2018 Gupta AK, et al. This is an open-access article distributed under the terms of the Creative Commons Attribution License, which permits unrestricted use, distribution, and reproduction in any medium, provided the original author and source are credited. 
aspect of image recognition and pattern detection. The issue was that we need to attach the Zoomer Interface with the trained model to make the machine capable to detect the blurred and tiny objects in the captured image [4]. The benefits and advantages which is provided by this model are:

- High Accuracy use of deep learning algorithm with highest accuracy on market.

- Simple setup and use implement cutting edge vision automation faster with low development costs.

- Recognize anything Train custom neural network to recognize your specific images.

- Training interface Create powerful and custom image recognizers in intuitive web interface.

- Scalable scale up with low infrastructure costs.

- Always improving the machine algorithms so everyone is up to date.

The working of Renshaw model is categorised as:

Web platform: Firstly, user provides the samples of images which are required to recognize. After providing the sample then the machine does cloud processing and send to the server where the images are tested, and model is trained and then stored. The result has provided to the user within 30 minutes and then the user can provide the feedback (Figure 3).

Mobile platform: Mobile Platform uses the technology stack and the cloud is promptly used and defined. Single image processing fires 8 billion floating point operations. Each model has 25 million of parameters. Neural network now trains 50 million images within 30 minutes. Each GPU provides 5 TFLOPS of processing power. These are some of the major changes which are engloted with the inclusion of technology cloud stack (Figures 4 and 5).

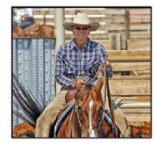

1. Input image

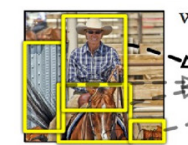

2. Extract region proposals $(\sim 2 \mathrm{k})$

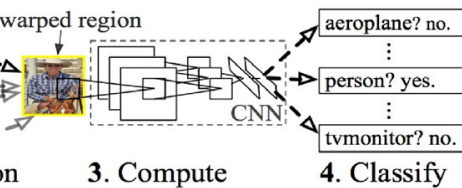

$\mathrm{CNN}$ features

Figure 1: After creating a set of region proposals, R-CNN passes the image through a modified version of Alex Net to determine whether or not it is a valid region.

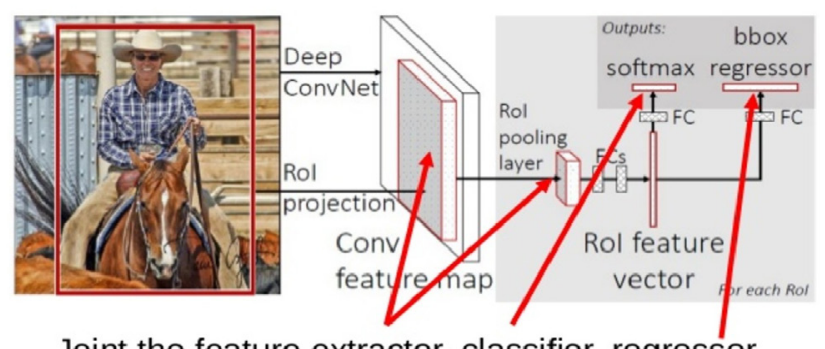

Joint the feature extractor, classifier, regressor together in a unified framework

Figure 2: Framework combined the $\mathrm{CNN}$, classifier and bounding box regressor into one.

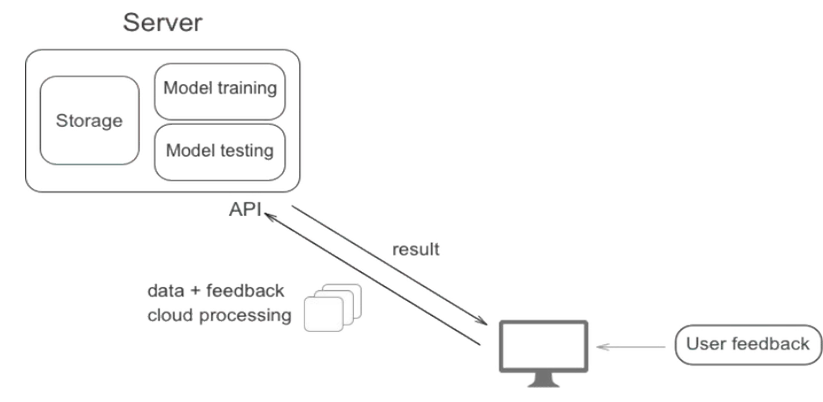

Figure 3: Web Platform Execution Loop.

When we talk about the cloud usage than we are talking about the flexibility which has given to clients or the users for making the technology being understand more easily.

\section{Algorithms}

\section{Regression tree}

Regression Trees are an important type of algorithm for predictive modelling machine learning. The representation of the decision tree model is a binary tree. This is your binary tree from algorithms and data structures, nothing too fancy. Each node represents a single input variable $(\mathrm{x})$ and a split point on that variable (assuming the variable is numeric) [5]. The leaf nodes of the tree contain an output variable (y) which is used to make a prediction. Predictions are made by walking the splits of the tree until arriving at a leaf node and output the class value at that leaf node. Trees are fast to learn and very fast for making predictions. They are also often accurate for a broad range of problems and do not require any special preparation for your data.

\section{Linear discriminant analysis}

Logistic Regression is a classification algorithm traditionally limited to only two-class classification problems. If you have more than two classes, then the Linear Discriminant Analysis algorithm is the preferred linear classification technique. The representation of LDA is pretty straight forward. It consists of statistical properties of your data, calculated for each class. For a single input variable this includes: The mean value for each class and the variance calculated across all classes. Predictions are made by calculating a discriminate value for each class and making a prediction for the class with the largest value. The technique assumes that the data has a Gaussian distribution (bell curve), so it is a good idea to remove outliers from your data beforehand. It's a simple and powerful method for classification predictive modelling problems.

\section{Linear vector quantization}

A downside of K-Nearest Neighbours is that you need to hang on to your entire training dataset. The Learning Vector Quantization algorithm (or LVQ for short) is an artificial neural network algorithm that allows you to choose how many training instances to hang onto and learns exactly what those instances should look like (Figure 6).

The representation for LVQ is a collection of codebook vectors. These are selected randomly in the beginning and adapted to best summarize the training dataset over a number of iterations of the learning algorithm. After learned, the codebook vectors can be used to make predictions just like KNearest Neighbours. The most similar neighbour (best matching codebook vector) is found by calculating the distance between each codebook vector and the new data instance. 


\section{Boosting}

Boosting is an ensemble technique that attempts to create a strong classifier from a number of weak classifiers. This is done by building a model from the training data, then creating a second model that attempts to correct the errors from the first model. Models are added until the training set is predicted perfectly, or a maximum number of models are added.

The main advantage of using the Boosting algorithm is if we are training the complex image constituting of more than one class than the one class is trained, and the object is added to the retrieval segment for making again the data to be trained.

\section{Use Cases}

Use cases are categorised in four categories:

\section{Specific}

Automate visual routines. Power innovative applications. Maximum flexibility and customization. It covers healthcare, science and research, manufacturing, agriculture, technical inspection (Figure 7).

\section{E-commerce}

Fine -grained categorization. Process millions of images. Abstract understanding. Recognize and categorize what you need. It covers fine - grained catalogue, content management automation, abstract understanding (Figure 8).

\section{Internet of things}

Power smart IoT applications. Automate routine monitoring.

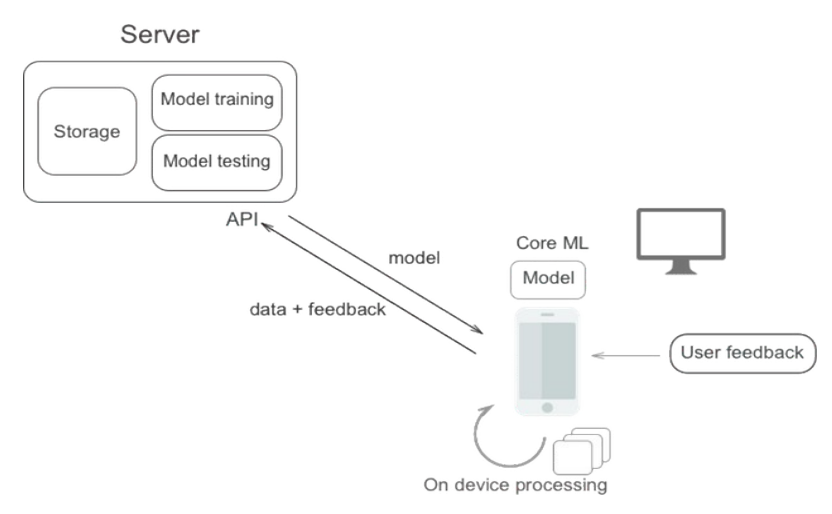

Figure 4: Mobile Platform Execution Loop.

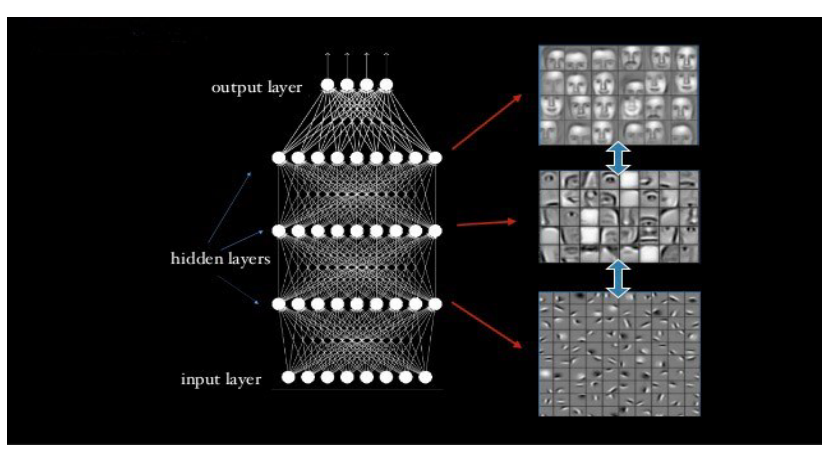

Figure 5: Technology Stack.

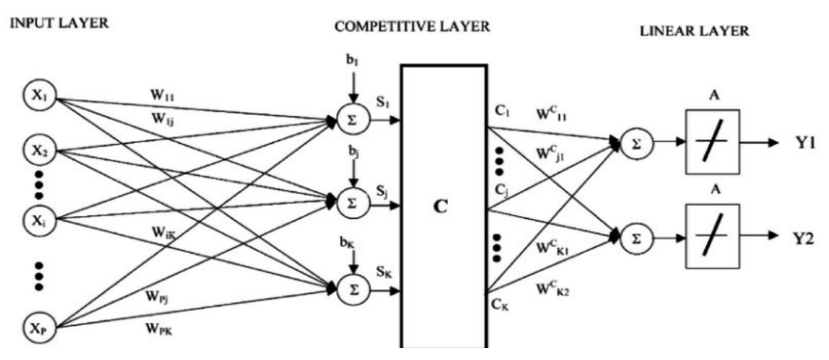

Figure 6: LVQ works as Map Reducer.

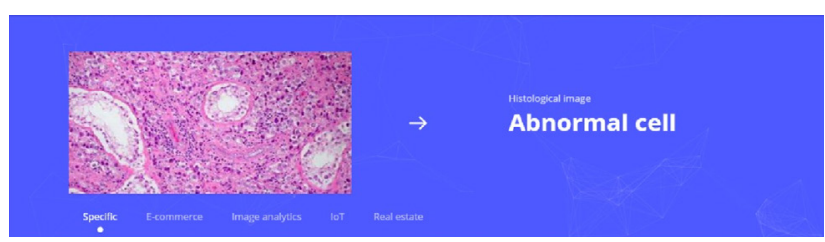

Figure 7: Specific

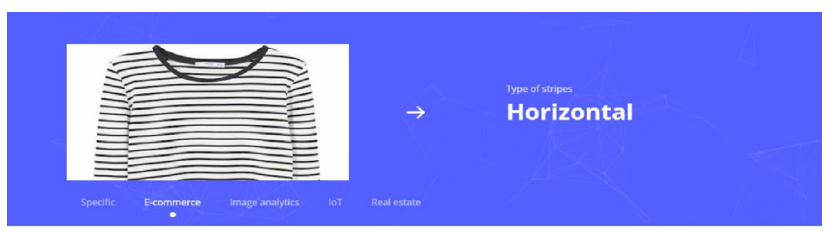

Figure 8: E-Commerce.

Bring intelligence to current hardware. It covers smart cameras images, drone monitoring, next generation of smart machines (Figure 9).

\section{Real estate}

Automate image processing. Enables detailed filtering. Enhance real - estate listings. It covers feature recognition, home scenes (Figure 10) $[6,7]$.

\section{Vize-Web Neural Artificial Platform}

The web platform which is designed on the concept used by the IITs to have the virtual labs and provide the fruitful knowledge among the children of each university. So, two top organisations planned to make the virtual web platform for making the students and clients understand the technology of machine learning and neural network easier and making the working life of the users simple.

The working of web platform is as follows:

- Client will be first required to send the request for using the software for what purpose.

- After receiving the mail, we will take 2-3 days to make decision and if the decision is yes than you will be getting the mail with E-Mail and password for working.

- When the client login it is required to identify maximum two classes.

- Two classes should have maximum 20 images per classes. If you do not meet the requirement of 20 images,

- i.e., 40 images in one go than you will not allowed to proceed further. 
- After uploading 20 complex images for each class; click on TRAIN button (Figure 11).

- Wait for minimum 30 minutes to proceed and you will receive mail that your classes are trained. After that refresh browser and check by identify any image you want for result.

The usage of this platform provides the demolishing of In- house development and moving towards the cloud services. The Network as a Service was not provided due to the security and providing the hundred percent accuracy with the diminished time complexity with iicreator tool for annotating the detected objects (Figure 12). In - house development covers specific scenarios. There is no data transfer and high development time and costs required. Whereas when we talk about SaaS it just made the machine learning very easy. Feedback loop and big data. Problem overview and the concept of optimization and Meta learning. Infrastructure and scaling with low costs.

Most of the machine learning applications are designed to improve in time based on a data feedback loop. Machine learning flow is based on new generated data and feedback for the current model performance. Model is periodically retrained to reflect the most relevant data [8].

\section{Conclusion}

The outcome of it will be as image to be as an input which will be checked whether it is motion object or not? If it is yes than it will be move for object tracking followed by the morphology process. Morphology process provides the input image to be contaminated

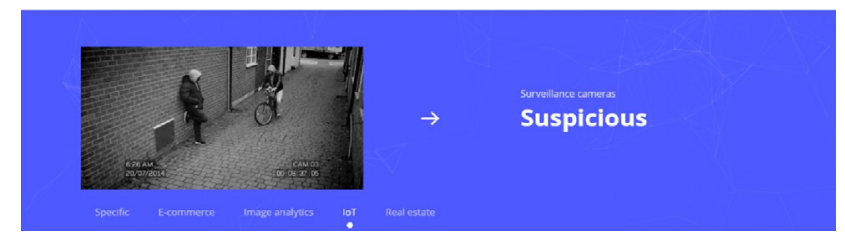

Figure 9: loT.

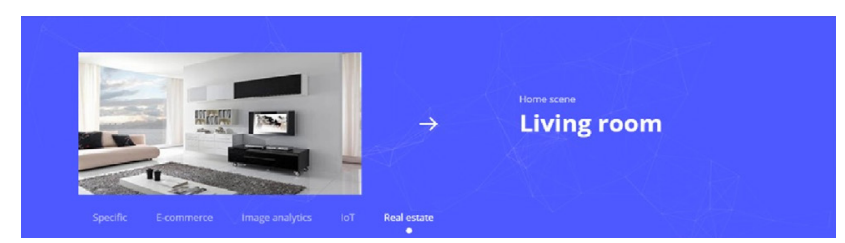

Figure 10: Real Estate.

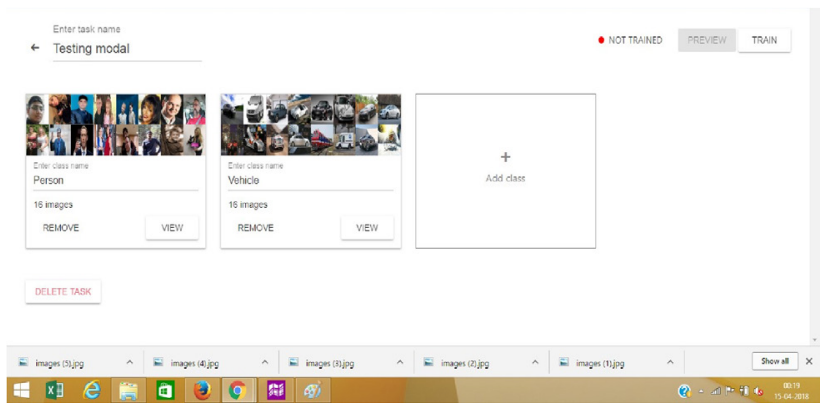

Figure 11: If the user training the class with less than 20 images than the "Train" option will not respond.

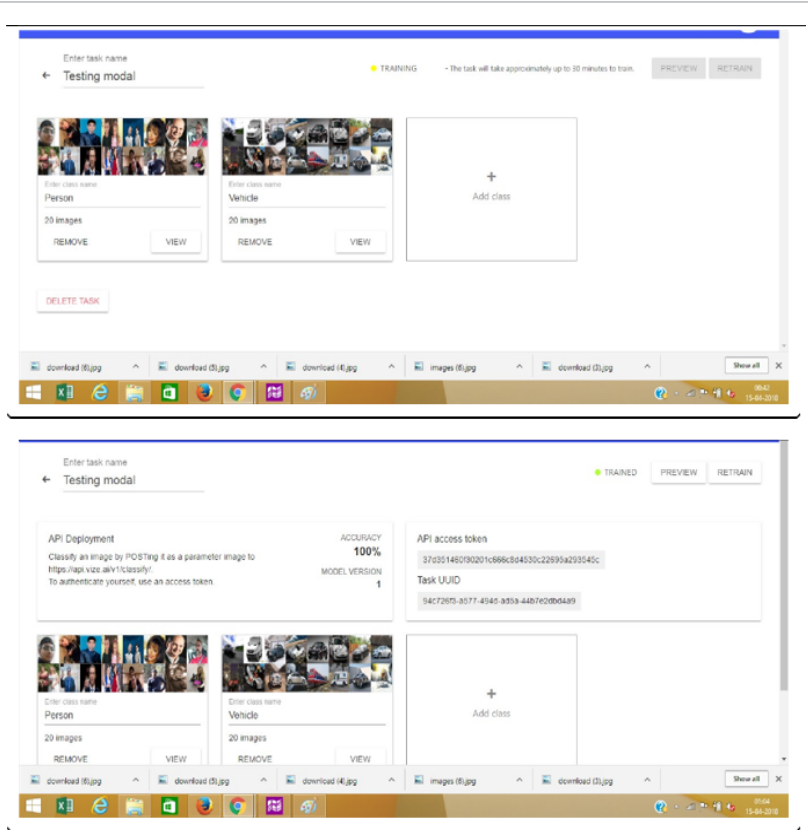

Figure 12: Two classes trained under specific use case.

with the boundary box and each individual object will be identified. In addition to this the token id will be provided to each individual user to train their own model. In addition to it we are providing the cloud storage so that memory usage could be safe.

\section{Acknowledgments}

During this ongoing research I was lucky to have such a supportive partner who helped me a lot in mathematical calculation. With the heroics of the depth knowledge inbuilt I would like to thank Mr Manpreet Sehgal who helped in letting us understand the domain knowledge, Dr. Vikas Jindal for helping us understanding the Automate system through Automata Theory, Dr. Sudhakar Ranjan who believes in us and provides us support and always standing as our backbone to remove all hurdles, Mrs. Deepti Thakral for letting us go through the new technologies which helps us in live stream convention. In the end I would like to thank my mother and God for their showering blessings and also Ms. Alinka Ayrapartyan for always supporting and understanding me and having trust with such a dedication. Thanks to all for believing in us.

\section{References}

1. Medium (2018) A Brief History of CNNs in Image Segmentation: From R-CNN to Mask R-CNN.

2. Vita Vaika: Research methodologies of Artificial Intelligence. London Campus UK.

3. Baecker RM, Grudin J, Buxton WAS, Greenberg S (1995) Readings in humancomputer interaction. Toward the Year 2000. 2nd edn. Morgan Kaufmann, San Francisco, USA, p: 950.

4. Haralick RM, Shanmugam K, Dinstein I (1973) Textural Features for Image Classification. IEEE Transactions on Systems, Man and Cybernetics 3: 610621.

5. Lin M, Chen Q, Yan S (2014) Network in network. International Conference on Learning Representations. Banff, Canada.

6. Lin T, Maire M, Belongie S, Bourdev L, Girshick R, et al. (2014) Microsoft COCO: common objects in context. arXiv e-prints.

7. Simonyan K, Zisserman A (2015) Very deep convolutional networks for large-scale image recognition. International Conference on Learning Representations Banff, Canada.

8. Viola P, Jones M (2001) Rapid object detection using a boosted cascade of simple features. IEEE Computer Society Conference on Computer Vision and Pattern Recognition, Kauai, HI, USA. 